\title{
A Passivity Approach to Controller-Observer Design for Robots
}

\author{
Harry Berghuis, Student Member, IEEE, and Henk Nijmeijer, Senior Member, IEEE
}

\begin{abstract}
Passivity-based control methods for robots, which achieve the control objective by reshaping the robot system's natural energy via state feedback, have, from a practical point of view, some very attractive properties. However, the poor quality of velocity measurements may significantly deteriorate the control performance of these methods. In this paper we propose a design strategy that utilizes the passivity concept in order to develop combined controller-observer systems for robot motion control using position measurements only. To this end, first a desired energy function for the closed-loop system is introduced, and next the controller-observer combination is constructed such that the closed-loop system matches this energy function, whereas damping is included in the controllerobserver system to assure asymptotic stability of the closed-loop system. A key point in this design strategy is a fine tuning of the controller and observer structure to each other, which provides solutions to the output-feedback robot control problem that are conceptually simple and easily implementable in industrial robot applications. Experimental tests on a two-DOF manipulator system illustrate that the proposed controller-observer systems enable the achievement of higher performance levels compared to the frequently used practice of numerical position differentiation for obtaining a velocity estimate.
\end{abstract}

\section{INTRODUCTION}

$\mathrm{T}$ HE NONLINEAR nature of the tracking control problem of robots has made this problem a challenging one in robotics research. The research efforts that evolved from this have yielded several solutions to the problem, of which the computed torque controller [1] is currently the best known. This controller achieves the tracking objective using the well-known nonlinear system theory concept of feedback linearization [2]. This illustrates that this method actually stems from a general system theoretic methodology, thereby neglecting the natural structure imposed by the physical character of the robot system.

In recent years, the so-called passivity-based approach [3] to robot control has gained much attention which, contrary to computed torque control, tackles the robot control problem by exploiting the robot system's physical struc-

Manuscript received April 27, 1992; revised November 4, 1992. H. Berghuis was supported by The Netherlands Technology Foundation (STW). H. Berghuis is with the Control Group, Department of Electrical En gineering, Mechatronics Research Center Twente, University of Twente, 7500 AE Enschede, The Netherlands.

H. Nijmeijer is with the Systems and Control Group, Department of Applied Mathematics, Mechatronics Research Center Twente, University of Twente, $7500 \mathrm{AE}$ Enschede, The Netherlands.

IEEE Log Number 9212609 ture. The idea of this controller design philosophy is to reshape the robot system's natural energy such that the control objective is achieved. This is done by constructing the controller to meet a desired energy function for the closed-loop system, and add damping, via velocity feedback, for asymptotic stabilization purposes [4]. Since in this way the passivity property of robots is preserved in the closed loop, this idea has been designated as passivity approach [3]. Based on this philosophy, Takegaki and Arimoto [5] proposed a beautiful, simple solution to the robot position control problem, whereas its natural extension to the motion control task was solved by Paden and Panja [6]. Also, for adaptive robot control the passivitybased approach has been studied extensively, see e.g., [7]-[9].

Passivity-based schemes are all characterized by a linear state feedback part (frequently referred to as proportional-derivative (PD) feedback), which is in fact inherent to the underlying design philosophy. This shows that these schemes are close to the classical design approaches. In addition, these controllers tend to be robust [4], and can provide attractive shortcuts on the computational level, which can be seen particularly from a comparison of the position control scheme of Takegaki and Arimoto [5] and the computed torque solution to position control. These properties let us conclude that the passivity-based robot control methods could be very attractive from a practical point of view.

Unfortunately, the need for velocity feedback shows one drawback of both computed torque and passivitybased control methods since in practice this can only be partially fulfilled for two reasons. First, although robot systems are generally equipped with high precision sensors for position measurements, velocity measurements, e.g., by tachometers, are often contaminated with a considerable amount of noise. As this imparts severe limitations on the attainable closed-loop bandwidth, it may significantly deteriorate the control performance [10], [11]. Second, in robotic applications today velocity sensors are frequently omitted due to the considerable savings in cost, volume, and weight that can be obtained in this way [12].

One way to remedy the velocity feedback problem is to determine a velocity signal by first-order numerical differentiation of the accurate position signal. The simplicity of this technique makes it particularly useful from an implementation point of view. However, for low and high 
velocities especially, such a simple approximation of the velocity signal may be inadequate [13], [14]. Moreover, the quantization effect that inherently goes along with this approach may produce undesired oscillations in the robot joint response, or even cause it to become unstable [12]. In addition, there is no theoretical justification for this $a d$ hoc solution, i.e., closed-loop stability properties have not been established.

A more sophisticated approach to solve the velocity feedback problem is the design of an observer that utilizes the high-quality position information for reconstructing the velocity signal. Although the nonlinear structure of the robot system makes this observer design problem a complex one, some solutions have already been proposed, for example based on the sliding mode concept [15], [16], and on a linear high-gain strategy [17]. These observers are universally applicable in the sense that they have been developed independent of the robot control scheme for which the velocity estimate is generated. However, an observer that asymptotically reconstructs the state of a nonlinear system does not guarantee that a given stabilizing state-feedback controller will remain stable when using the estimated state instead of the true one in the feedback loop; in general a nonlinear separation principle is not valid.

This stability problem has motivated the design of combined controller-observer schemes for robot systems using position feedback only. For example, Canudas de Wit et al. [18] propose a modified version of the computedtorque controller in which the actual velocity is replaced by an estimate obtained from a nonlinear observer, and prove local exponential stability of the overall system under some suitable conditions. Also Nicosia and Tomei [19] consider some known state-feedback controllers with a nonlinear observer inserted in the feedback loop, and guarantee local asymptotic stability. Besides the stability argument, however, it is important to observe that in a combined controller-observer design approach one may be able to efficiently tune the controller and observer structure to each other. This point is illustrated by the combined computed torque controller-linear observer idea of Berghuis et al. [20], in which the linear observer exploits the feedback linearizing property of the computed torque controller. In this paper we will show how this key idea of tuning can be applied to passivity-based robot control.

In particular we will present a systematic procedure that utilizes the passivity concept in order to design combined robot controller-observer systems for both the positioning and tracking problem. For this purpose we will introduce a desired energy function that consists of two parts, one that accounts for the closed-loop controller dynamics, and another part for the closed-loop observer dynamics. Next, via some design examples we will illustrate how to construct combined controller-observer systems such that for the closed-loop system the desired energy relation is established, whereas by the inclusion of damping in the control system we will assure asymptotic stability of the closed-loop under some suitable conditions on the controller and observer gains. The control methods developed in this way will be referred to as passivity-based output-feedback robot controllers.

The organization of this paper is as follows. The next section presents the design strategy, which in Section III will be used in order to develop some passivity-based output-feedback robot controllers. In Section IV the results of an experimental study towards performance and robustness properties of one of the presented control methods will be presented. We will end with some concluding remarks.

\section{Design Strategy for Passivity-Based Output- FEEDBaCK CONTROL}

\section{A. Introduction to the Passivity Approach to Robot Control}

Consider the standard equations describing the dynamics of an $n$-DOF rigid robot system

$$
M(q) \ddot{q}+C(q, \dot{q}) \dot{q}+G(q)=\tau
$$

where $M(q)$ is the positive definite inertia matrix $[n \times n]$, $C(q, \dot{q}) \dot{q}$ the Coriolis and centrifugal torques $[n \times 1]$, $G(q)$ the gravitational torques $[n \times 1]$, and $\tau$ the control input $[n \times 1]$. These motion equations possess several properties that can be exploited to facilitate the controller design, see for instance [3]. For future reference these properties are shortly recalled here.

Property 2.1: The class of rigid robot systems is passive from $\tau$ to $\dot{q}$, that is

$$
\langle\dot{q}, \tau\rangle \equiv \int_{0}^{t} \dot{q}^{T}(s) \tau(s) d s \geq-\beta
$$

where $\beta=H_{0}(q(0), \dot{q}(0))>0$, and $H_{0}(q, \dot{q})$ the total (co-)energy of the system (1), i.e.

$$
H_{0}(q, \dot{q})=\frac{1}{2} \dot{q}^{T} M(q) \dot{q}+P(q)
$$

with $P(q)$ the potential energy. $P(q)$ is assumed to have an absolute minimum at $q=0$.

Property 2.2: By defining $C(q, \dot{q})$ using the Christoffel symbols, $\dot{M}(q)-2 C(q, \dot{q})$ is skew-symmetric. Moreover, for this choice $C(q, \dot{q})$ satisfies

$$
\begin{aligned}
C(q, x) y= & C(q, y) x \quad \text { for all } x, y \\
C(q, z+\alpha x) y= & C(q, z) y+\alpha C(q, x) y \\
& \text { for all } x, y \quad \alpha \text { scalar. }
\end{aligned}
$$

Property 2.3: For revolute robots the matrices $M(q)$ and $C(q, \dot{q})$ are bounded w.r.t. $q$, so

$$
\begin{aligned}
& 0<M_{m} \leq\|M(q)\| \leq M_{M} \quad \text { for all } q \\
&\|C(q, x)\| \leq C_{M}\|x\| \quad \text { for all } q, x
\end{aligned}
$$

where in (5) and in the sequel we define the norm of a vector $x$ as

$$
\|x\|=\sqrt{x^{T} x}
$$


and the norm of a matrix $A$ as

$$
\|A\|=\sqrt{\lambda_{\max }\left(A^{T} A\right)}
$$

with $\lambda_{\max }(\cdot)$ the maximum eigenvalue. Moreover, for any matrix $A(x)=A^{T}(x)>0$ and for all $x, A_{m}$, and $A_{M}$ denote the minimum and maximum eigenvalue of $A(x)$, respectively.

To provide the background for our work, we will shortly recapitulate the ideas underlying the passivitybased control methodology. To this end, assume that the control objective consists of positioning the robot at some desired position $q_{d}$. It is intuitively clear that this can be achieved by shifting the strict energy minimum at $(q, \dot{q})$ $=(0,0)$ to $(e, \dot{q})=(0,0)$, where $e \equiv q-q_{d}$ represents the position error. According to (3), this shifting can be realized by reshaping the potential energy of the system such that it obtains the desired minimum. To illustrate this, define the control law as (cf. Takegaki and Arimoto [5])

$$
\tau=G(q)-K_{p} e+\nu
$$

where $\nu$ represents a new control input. This choice of the control law actually modifies the original mechanical energy function (3) of the open-loop system (1) into

$$
H_{1}(e, \dot{q})=\frac{1}{2} \dot{q}^{T} M(q) \dot{q}+\frac{1}{2} e^{T} K_{p} e
$$

for which it can easily be verified that

$$
\dot{H}_{1}(e, \dot{q})=\dot{q}^{T} \nu \text {. }
$$

This implies that a marginally stable closed-loop system is obtained [4] that is passive from the new input $\nu$ to $\dot{q}$, since

$$
\langle\dot{q}, \nu\rangle \equiv \int_{0}^{t} \dot{q}^{T}(s) \nu(s) d s \geq-H_{1}(e(0), \dot{q}(0)) .
$$

To stabilize the system, damping should be added, so choose

$$
\nu=-K_{d} \dot{q} .
$$

Then LaSalle's invariance principle can be employed to complete the asymptotic stability proof.

For tracking purposes it is intuitively clear that the controller should be constructed such that the minimum ( $q$, $\dot{q})=(0,0)$ of the open-loop is shifted towards $(e, \dot{e})=$ $(0,0)$ for the closed loop. To attain this objective, Paden and Panja [6] propose the motion controller

$$
\begin{aligned}
& \tau=M(q) \ddot{q}_{d}+C(q, \dot{q}) \dot{q}_{d}+G(q)-K_{p} e+\nu \\
& \nu=-K_{d} \dot{e} .
\end{aligned}
$$

This controller structure establishes a passive mapping between $\nu$ and $\dot{e}$, as can be verified by evaluating the timederivative of the energy function

$$
H_{1}(e, \dot{e})=\frac{1}{2} \dot{e}^{T} M(q) \dot{e}+\frac{1}{2} e^{T} K_{p} e
$$

along (1), (13a). To prove asymptotic error convergence, Paden and Panja applied a theorem of Matrosov, see [6]. It is important to observe that (13) is a natural extension of the seminal result (8), (12) of Takegaki and Arimoto [5].

A different passivity-based controller was presented by Slotine and Li [8]. Their idea, which originates from sliding mode theory, has constituted a major breakthrough in adaptive control of robots. In particular, Slotine and $\mathrm{Li}$ [8] propose the tracking controller (cf. [21])

$$
\begin{aligned}
& \tau=M(q) \ddot{q}_{r}+C(q, \dot{q}) \dot{q}_{r}+G(q)-K_{p} e+\nu \\
& \nu=-K_{d} s_{1}
\end{aligned}
$$

where

$$
\begin{aligned}
& \dot{q}_{r} \equiv \dot{q}_{d}-\Lambda_{1} e \\
& s_{1} \equiv \dot{q}-\dot{q}_{r}=\dot{e}+\Lambda_{1} e
\end{aligned}
$$

and $\Lambda_{1}=\Lambda_{1}^{T}>0, K_{d}=K_{d}^{T}>0$, and $K_{p}=K_{p}^{T}>0$. For simplicity here and in the sequel we will assume the following.

Assumption 2.1: $K_{p}, K_{d}$, and $\Lambda_{1}$ are constant and diagonal.

In this case, the closed-loop (1), (15a) defines a (strictly) passive mapping from $\nu$ to $s_{1}$, as can be verified by evaluating the time derivative of the modified energy function

$$
H_{1}\left(e, s_{1}\right)=\frac{1}{2} s_{1}^{T} M(q) s_{1}+\frac{1}{2} e^{T} K_{p} e
$$

along (1), (15a), i.e.,

$$
M(q) \dot{s}_{1}+C(q, \dot{q}) s_{1}+K_{p} e=\nu .
$$

To establish stability, note that by virtue of $(15 \mathrm{~b})$, the time derivative of (18) becomes

$$
\dot{H}_{1}\left(e, s_{1}\right)=-s_{1}^{T} K_{d} s_{1}-e^{T} \Lambda_{1} K_{p} e .
$$

Then from (18), (20) global asymptotic stability can be guaranteed.

The foregoing illustrates that the passivity approach to robot control consists of constructing a controller such that the closed-loop system matches a desired energy function that resembles the natural energy contents of the openloop system. In this way, passivity of the robot system can be preserved in the closed loop, for which reason the concept was designated as passivity based [3]. Moreover, by the inclusion of damping in the loop via velocity feedback, asymptotic stability can be attained. A for the realm of applications interesting feature of this concept is that it yields computationally attractive controllers that can be considered as extensions of the classical PD approach, see e.g., (13).

Also alternative solutions to the robot motion control problem based on this passivity concept have been developed, e.g. [22], [23]. The important observation is, however, that all these results are based on desired energy functions that fit into the following general formulation

$$
H_{1}\left(e, s_{1}\right)=\frac{1}{2} s_{1}^{T} M(q) s_{1}+\frac{1}{2} e^{T} K_{1}(q, e) e
$$

where $K_{1}(q, e)=K_{1}^{T}(q, e)>0, s_{1}=\dot{e}+\Lambda_{1} e$, and $\Lambda_{1}$ $=\Lambda_{1}^{T}>0$ not necessarily constant. The actual choices 
for $s_{1}$ and $K_{1}(q, e)$ that have been considered in the literature differ in an interesting way (see e.g. , [3], [6], [8], $[21],[23])$, and are in fact strongly related to the proof technique used in the stability analysis.

Remark 2.1: The key idea in the scheme (15) is the introduction of the sliding variable $s_{1}$. This variable allows to straightforwardly conclude global asymptotic stability of the closed-loop system (1), (15), without the need for invoking advanced stability techniques such as in [6]. As a consequence, however, (15) does not possess what will be defined as the reduction property. That is, for position control (15) does not reduce to a simple PD with gravitation compensation, see $(8),(12)$, but two additional terms are present: $-M(q)\left(\Lambda_{1} \dot{e}\right)-C(q, \dot{q})\left(\Lambda_{1} e\right)$. This implies that in regulation (15) still requires knowledge of inertial and centrifugal terms, unlike the controller (13) of [6].

\section{B. A Passivity Approach to Observer Design}

In this section the velocity observer design problem for rigid robot systems is studied as a support for our further developments. This problem consists of constructing an auxiliary dynamic system that asymptotically reconstructs the velocity signal from input-output measurements, i.e., torque $\tau$ and position $q$, respectively. A new approach to this problem is proposed that is inspired by the passivitybased controller design concept. Corresponding to this concept, the problem is tackled by constructing an observer that forces the observer error dynamics to match a desired energy function, thereby preserving the passivity property. In addition, for asymptotic stabilization purposes damping has to be included in the loop. An interesting feature of this new approach is that it establishes a striking conceptual symmetry, or conceptual duality, between the strategies to robot controller and observer design. Such a duality feature is rather unique for nonlinear systems.

To illustrate the proposed approach, rewrite the robot dynamics (1) in state-space form

Robot system $\left\{\begin{array}{l}\frac{d}{d t} q=\dot{q} \\ \frac{d}{d t} \dot{q}=M^{-1}(q)[\tau-C(q, \dot{q}) \dot{q}-G(q)] .\end{array}\right.$

Based on this physical structure, introduce the general velocity observer system

$$
\text { Observer } \begin{aligned}
& \frac{d}{d t} \hat{q}=z+L_{d} \hat{q} \\
& \frac{d}{d t} z= M^{-1}(q)\left(\tau-C\left(q, \dot{q}_{0}\right) \dot{q}_{0}\right. \\
&\left.-G(q)+L_{p 1} \tilde{q}\right)+L_{p 2} \tilde{q}
\end{aligned}
$$

where $\left[\hat{q}^{T} z^{T}\right]$ is the observer state, $\dot{\hat{q}}$ represents the estimated velocity, $\tilde{q} \equiv q-\hat{q}$ is the observer position estimation error, $L_{d}=L_{d}^{T}>0, L_{p 1}=L_{p 1}^{T} \geq 0, L_{p 2}=L_{p 2}^{T}>$ 0 , and

$$
\dot{q}_{0}=\dot{\hat{q}}-\Lambda_{2} \tilde{q}
$$

with $\Lambda_{2}=\Lambda_{2}^{T} \geq 0$ can be considered the dual of $\dot{q}_{r}$ in (16)

Assumption 2.2: $L_{d}, L_{p 1}, L_{p 2}$, and $\Lambda_{2}$ are constant and diagonal. Moreover, $L_{d}$ and $L_{p 2}$ can be written as

$$
\begin{aligned}
L_{d} & =l_{d} I+\Lambda_{2} \\
L_{p 2} & =l_{d} \Lambda_{2}
\end{aligned}
$$

where $l_{d}>0$ scalar.

In addition, in this section the velocity of the robot system is assumed to be bounded.

Assumption 2.3: The velocity signal $\dot{q}$ is bounded by $V_{P}$, i.e.,

$$
V_{P}=\sup _{t}\|\dot{q}(t)\|
$$

Under Assumption 2.2 the observer error dynamics (1)(23) equals

$$
\begin{aligned}
& M(q) \dot{s}_{2}+C(q, \dot{q}) s_{2}+l_{d} M(q) s_{2}+L_{p 1} \tilde{q} \\
& =C\left(q, s_{2}\right)\left(s_{2}-\dot{q}\right)
\end{aligned}
$$

where

$$
\begin{aligned}
s_{2} & \equiv \dot{q}-\dot{q}_{0} \\
& =\dot{\tilde{q}}+\Lambda_{2} \tilde{q} .
\end{aligned}
$$

The following can be proved.

Proposition 2.1: Consider the observer (23) under Assumptions 2.2 and 2.3. Under the condition

$$
l_{d}>M_{m}^{-1} C_{M} V_{P}
$$

the error dynamics (1), (23) are locally exponentially stable, that is, there exist constants $m, \rho>0$ such that

$$
\|x(t)\|^{2} \leq m e^{-\rho t}\|x(0)\|^{2} \quad t \geq 0
$$

where $x^{T}=\left[\begin{array}{ll}s_{2}^{T} & \tilde{q}^{T}\end{array}\right]$. Moreover, a region of attraction is given by

$$
B=\left\{x \in \mathbb{R}^{2 n} \mid\|x\|<\left(l_{d} M_{m} C_{M}^{-1}-V_{P}\right) \sqrt{\frac{P_{m}}{P_{M}}}\right\}
$$

where $P_{m}$ and $P_{M}$ are defined as

$$
P_{m}=\min \left\{M_{m}, L_{p 1, m}\right\}, P_{M}=\max \left\{M_{M}, L_{p 1, M}\right\} \text {. }
$$

Proof: Consider the desired energy relation

$$
H_{2}\left(\tilde{q}, s_{2}\right)=\frac{1}{2} s_{2}^{T} M(q) s_{2}+\frac{1}{2} \tilde{q}^{T} L_{p 1} \tilde{q} .
$$


The time-derivative of (33), evaluated along (27), becomes

$$
\begin{aligned}
\dot{H}_{2}\left(\tilde{q}, s_{2}\right)= & -s_{2}^{T} l_{d} M(q) s_{2}-\tilde{q}^{T} \Lambda_{2} L_{p 1} \tilde{q} \\
& +s_{2}^{T} C\left(q, s_{2}\right)\left(s_{2}-\dot{q}\right) .
\end{aligned}
$$

Using Property 2.3 and Assumption 2.3, an upper bound on (34) is given by

$$
\begin{aligned}
\dot{H}_{2}\left(\tilde{q}, s_{2}\right) \leq & -\left(l_{d} M_{m}-C_{M}\left(\left\|s_{2}\right\|+V_{P}\right)\right)\left\|s_{2}\right\|^{2} \\
& -\Lambda_{2, m} L_{p 1, m}\|\tilde{q}\|^{2} .
\end{aligned}
$$

Take $V(x)=H_{2}\left(\tilde{q}, s_{2}\right)$, where $H_{2}\left(\tilde{q}, s_{2}\right)$ as defined in (33), as a Lyapunov function. This function satisfies

$$
\frac{1}{2} P_{m}\|x\|^{2} \leq V(x) \leq \frac{1}{2} P_{M}\|x\|^{2}
$$

where $P_{m}, P_{M}$ as defined in (32). Equation (35) shows that if

$$
\left\|s_{2}\right\|<l_{d} M_{m} C_{M}^{-1}-V_{p}
$$

then there exists a constant $\kappa>0$ such that

$$
\dot{V}(x) \leq-\kappa\|x\|^{2}
$$

for all $x$ satisfying (37), where the right-hand side of (37) is positive by (29). So (30) can be concluded from (36) and (38), which completes the first part of the proof.

Next, a sufficient condition for (37) to hold is

$$
\|x\|<l_{d} M_{m} C_{M}^{-1}-V_{P}
$$

which implies that if

$$
\|x(0)\|<\left(l_{d} M_{m} C_{M}^{-1}-V_{P}\right) \sqrt{\frac{P_{m}}{P_{M}}}
$$

then from (36), (38), (39)

$$
\begin{array}{ll}
V(x(t)) \leq V(x(0)) & \text { for all } t \geq 0 \\
\dot{V}(x(t)) \leq-\kappa\|x(t)\|^{2} & \text { for all } t \geq 0 .
\end{array}
$$

This completes the proof.

Clearly the exponential convergence of $x$ implies the exponential convergence of the natural observer error state $y^{T}=\left[\dot{\tilde{q}}^{T} \tilde{q}^{T}\right]$, because $x$ and $y$ are linearly related. Moreover, it is important to observe from (31), (32) that the attraction region grows $O\left(l_{d}\right)$, which means that for a given set of gains $L_{p 1}, \Lambda_{2}$, this region can be made arbitrarily large by increasing the observer gain $l_{d}$. In modern terminology this kind of stability is called semiglobal.

The particular structure of the observer gains $L_{d}$ and $L_{p 2}$, see Assumption 2.2, enables the introduction of the sliding variable $s_{2} \equiv \dot{\tilde{q}}+\Lambda_{2} \tilde{q}$ in the observer error dynamics (27). The meaning of this variable is dual to $s_{1} \equiv$ $\dot{e}+\Lambda_{1} e$ in the passivity-based control methodology that was discussed in Section II-A. The analogy between the passivity-based controller (15) and the observer (23) can also be seen by comparing the desired energy relations (18), (33), respectively, the error dynamics (15b), (19), (27), respectively, and the auxiliary signals $\dot{q}_{r}, \dot{q}_{0}$ in (16), (24), respectively. Unfortunately, the stability analysis for the observer is more elaborate than that for its control equivalent, which is due to the quadratic velocity dependence of the Coriolis and centrifugal terms. This introduces third-order terms in the Lyapunov derivative, which cannot be compensated for exactly due to the missing velocity signal, and as a consequence the result is only semiglobal. From the time-derivative (35) it can be seen that the third-order terms disappear if $C_{M} \equiv 0$, which confirms that the Coriolis and centrifugal torques cause the locality.

Passivity Preservation: The error dynamics (27) can be interpreted as a feedback interconnection of two subsystems (assume for simplicity $L_{p 1} \equiv 0$ ):

$$
\begin{gathered}
M(q) \dot{s}_{2}+C(q, \dot{q}) s_{2}=\nu \\
\nu=-l_{d} M(q) s_{2}+C\left(q, s_{2}\right)\left(s_{2}-\dot{q}\right) .
\end{gathered}
$$

For (42a) it can easily be verified that it is passive from $\nu$ to $s_{2}$, since

$$
\left\langle s_{2}, \nu\right\rangle \equiv \int_{0}^{T} s_{2}^{T}(s) \nu(s) d s \geq-H_{2}\left(\tilde{q}(0), s_{2}(0)\right)
$$

where $H_{2}\left(\tilde{q}, s_{2}\right)$ as in (33). This shows that the observer error dynamics preserves the passivity of (1), analog to the passivity methodology to controller design. Inspired by this, the constructed observer is baptized as passivitybased observer.

Besides, the block (42b) defines a strictly passive mapping from $s_{2}$ to $-\nu$, since

$$
\begin{aligned}
\left\langle-\nu, s_{2}\right\rangle & =-\int_{0}^{T} \nu^{T}(s) s_{2}(s) d s \\
& =\int_{0}^{T}\left\{s_{2}^{T} l_{d} M(q) s_{2}-\left(s_{2}-\dot{q}\right)^{T} C^{T}\left(q, s_{2}\right) s_{2}\right\} d s
\end{aligned}
$$

and under assumption (39)

$$
\left\langle-\nu, s_{2}\right\rangle \geq \kappa\left\|s_{2}\right\|_{2 T}^{2}
$$

where $\kappa>0$ constant and $\|\cdot\|_{2 T}^{2}$ the truncated $L_{2}$-norm. Equations (43) and (44) imply that $s_{2} \in L_{2}$, and the error proof can also be completed using the $I-O$-passivity tools discussed in [24] (see also [7] and [9]).

Remark 2.2: As referred to in [3], the passivity-based control methodology allows for variations by generalizing the definition of the sliding variable $s_{1}$. The observation problem allows for a similar generalization of the definition of $s_{2}$, that is

$$
\begin{aligned}
& \dot{q}_{0}=\dot{\hat{q}}-K_{2}(s) \tilde{q} \\
& s_{2}=F_{2}(s) \tilde{q}
\end{aligned}
$$


where $K_{2}(s)$ and $F_{2}(s)$ satisfy

$$
F_{2}(s)=s I+K_{2}(s)
$$

and $K_{2}(s)$ should be chosen such that $F_{2}^{-1}(s)$ is strictly proper and stable. For instance, assume

$$
K_{2}(s)=\sum_{i=0}^{k} \frac{\Lambda_{2 i}}{s^{i}}
$$

with $\Lambda_{2 i}>0$ diagonal, $i \in 0, \cdots, k$. In correspondence to this generalized definition of $s_{2}$, the observer system changes into

Observer $\left\{\begin{array}{l}\frac{d}{d t} \hat{q}=z_{0}+L_{d} \tilde{q} \\ \frac{d}{d t} z_{0}=z_{1}+L_{p 1} \tilde{q}+M^{-1}(q) \\ \frac{d}{d t} z_{1}=z_{2}+L_{p 2} \tilde{q} \\ \vdots \\ \frac{d}{d t} z_{k-1}=z_{k}+L_{p k} \tilde{q} \\ \frac{d}{d t} z_{k}=L_{p(k+1)} \tilde{q}\end{array}\right.$

where $L_{d}=l_{d} I+\Lambda_{20}, L_{p j}=l_{d} \Lambda_{2(j-1)}+\Lambda_{2 j}$ for $j=1$, $\cdots, k$, and $L_{p(k+1)}=l_{d} \Lambda_{2 k}$. Then under condition (29), semiglobal asymptotic error convergence of the closedloop system (1), (48) can be guaranteed along the same steps as the proof of Proposition 2.1, for an arbitrary $k$. Notice that to this end also the passivity theorem in [24] has to be invoked because the energy function (33) is not a strict Lyapunov function for the generalized definition (45b) of $s_{2}$. The actual choice of $k$ is determined by practical considerations such as disturbance rejection.

Remark 2.3: For the stabilizability proof of the openloop observer system (23) we need the boundedness of the velocity signal $\dot{q}$, which is a reasonable assumption from a practical point of view. Nevertheless, in the combined controller-observer design approach, see Sections II-C and III, this assumption is not required any more.

Remark 2.4: By choosing $\Lambda_{2} \equiv 0$ we obtain the openloop observer that is proposed by Nicosia and Tomei [19], which shows that (27) is actually a generalization of the Nicosia and Tomei observer.

\section{A Strategy for Combined Controller-Observer Design}

In Section II-A the principles that underlie the passivity-based methodology to robot control were highlighted.
Based on duality arguments, in Section II-B a similar approach was developed for the observer problem. As a consequence, for the design of passivity-based controller-observer systems it seems natural to take as a starting point a desired energy function that consists of a combination of the desired energy relations for the controller and observer system. This is also intuitively motivated by the fact that energy is an additive quantity. For this reason, consider the desired energy function

$$
\begin{aligned}
H_{3}\left(e, s_{1}, \tilde{q}, s_{2}\right)= & \frac{1}{2} s_{1}^{T} M(q) s_{1}+\frac{1}{2} e^{T} K_{1}(q, e) e \\
& +\frac{1}{2} s_{2}^{T} M(q) s_{2}+\frac{1}{2} \tilde{q}^{T} K_{2}(q, \tilde{q}) \tilde{q}
\end{aligned}
$$

where $K_{1}(q, e)=K_{1}(q, e)^{T}>0$ and $K_{2}(q, \tilde{q})=K_{2}(q$, $\tilde{q})^{T}>0$. A design strategy for passivity-based outputfeedback robot controllers, then consists of the following two steps.

1) Choose $s_{1}, K_{1}(q, e), s_{2}$ and $K_{2}(q, \tilde{q})$, in order to define a desired energy function that the closed-loop error dynamics should match. Construct a controller-observer combination in order to attain the desired energy relationship, and add "damping" to the system for asymptotic stabilization.

2) Use the desired energy relation as a Lyapunov function and determine the conditions on the controller and observer gains under which the time derivative of this Lyapunov function is negative definite. This guarantees asymptotic stability of the overall error system.

It is important to emphasize that for linear systems the above-mentioned strategy is greatly facilitated due to the separation principle [25], making the controller and observer design independent. For the class of nonlinear robot systems considered here, on the other hand, this principle is not valid. For this reason, a key point in the design of combined controller-observer systems for robots is a fine-tuning of the observer structure to the underlying control structure, as will be shown in Section III.

The proposed approach is based on the ability to construct a control system that establishes a desired energy function. It will be clear that the choice of this function is crucial because on the one hand it will not be possible to successfully complete the construction phase for an arbitrary choice of this function. On the other hand the desired energy relation largely fixes the controller-observer structure, so specific wishes concerning this structure such as the reduction property (see Remark 2.1) limit the class of desired energy functions.

Remark 2.5: As velocity is not measurable, we cannot introduce damping in the closed-loop system by velocity feedback. Therefore the injection of damping in the loop has now to be realized via the observer system. In Section III we will illustrate how this can be done.

Remark 2.6: It is interesting to recall recent work by 
Ortega and Espinosa [26]. These authors underscore the need of developing a system theoretic framework for control of nonlinear systems that incorporates at a fundamental level the system's physical structure and in particular its dissipation properties. Clearly, the above-mentioned approach would perfectly fit into such a framework.

\section{Some Illustrative Examples}

\section{A. The Slotine and Li Scheme for Output Feedback}

To illustrate the design strategy, the controller (15) is redesigned to a passivity-based output-feedback robot controller.

Step 1: In the case that only position information is available, $\ddot{q}_{r}$ in (16) cannot be realized. Therefore redefine

$$
\dot{q}_{r} \equiv \dot{q}_{d}-\Lambda_{1}\left(\hat{q}-q_{d}\right)
$$

and, consequently,

$$
s_{1}=\dot{q}-\dot{q}_{r} \equiv \dot{e}+\Lambda_{1}(e-\tilde{q}) .
$$

In addition, assume

$$
\dot{q}_{0} \equiv \dot{\hat{q}}-\Lambda_{2} \tilde{q}
$$

and, consequently,

$$
s_{2}=\dot{q}-\dot{q}_{0} \equiv \dot{\tilde{q}}+\Lambda_{2} \tilde{q} .
$$

The control law is chosen as

$$
\begin{aligned}
\tau= & M(q) \ddot{q}_{r}+C\left(q, \dot{q}_{0}\right) \dot{q}_{r}+G(q) \\
& -K_{d}\left(s_{1}-s_{2}\right)-K_{p} e
\end{aligned}
$$

where the observer signal $s_{2}$ in (54) is introduced because $s_{1}$ cannot be realized, whereas the difference $s_{1}-s_{2}$ is a function of known signals, i.e.,

$$
s_{1}-s_{2}=\left(\dot{\hat{q}}-\dot{q}_{d}\right)+\Lambda_{1}(e-\tilde{q})-\Lambda_{2} \tilde{q} .
$$

The closed-loop tracking error dynamics (1)-(54) becomes

$$
\begin{gathered}
M(q) \dot{s}_{1}+C(q, \dot{q}) s_{1}+K_{d} s_{1}+K_{p} e \\
=K_{d} s_{2}-C\left(q, s_{2}\right) \dot{q}_{r} .
\end{gathered}
$$

Next, the observer should be designed. For this purpose, consider the following choice:

$$
\begin{aligned}
& \dot{\hat{q}}=z+L_{d} \tilde{q} \\
& \dot{z}=\ddot{q}_{r}+M^{-1}(q)\left(L_{p 1} \tilde{q}-K_{p} e\right)+L_{p 2} \tilde{q}
\end{aligned}
$$

where $L_{d}$ and $L_{p 2}$ satisfy Assumption 2.2. Then the observer error dynamics equals

$$
M(q)\left(\dot{s}_{2}+l_{d} s_{2}\right)+L_{p 1} \tilde{q}=M(q) \dot{s}_{1}+K_{p} e
$$

which, using (56), can be rewritten as

$$
\begin{aligned}
& M(q) \dot{s}_{2}+C(q, \dot{q}) s_{2}+\left(l_{d} M(q)-K_{d}\right) s_{2}+L_{p 1} \tilde{q} \\
& =-K_{d} s_{1}+C\left(q, s_{1}\right)\left(s_{2}-\dot{q}\right) .
\end{aligned}
$$

Similar to the desired energy relation for the separate con- troller and observer, (18) and (33), assume that $K_{1}(q, e)$ $=K_{p}$ and $K_{2}(q, \tilde{q})=L_{p 1}$.

Step 2: The time-derivative of the desired energy function

$$
\begin{aligned}
H_{3}\left(e, s_{1}, \tilde{q}, s_{2}\right)= & \frac{1}{2} s_{1}^{T} M(q) s_{1}+\frac{1}{2} e^{T} K_{p} e \\
& +\frac{1}{2} s_{2}^{T} M(q) s_{2}+\frac{1}{2} \tilde{q}^{T} L_{p 1} \tilde{q}
\end{aligned}
$$

along the error-dynamics (56), (59) is given by

$$
\begin{aligned}
& \dot{H}_{3}\left(e, s_{1}, \tilde{q}, s_{2}\right) \\
&=-s_{1}^{T} K_{d}\left(s_{1}-s_{2}\right)-s_{2}^{T} l_{d} M(q) s_{2}+s_{2}^{T} K_{d}\left(s_{2}-s_{1}\right) \\
&+s_{1}^{T} C\left(q, s_{2}\right)\left(s_{1}-\dot{q}\right)+s_{2}^{T} C\left(q, s_{1}\right)\left(s_{2}-\dot{q}\right) \\
&-e^{T} \Lambda_{1} K_{p} e+\tilde{q}^{T} \Lambda_{1} K_{p} e-\tilde{q}^{T} \Lambda_{2} L_{p 1} \tilde{q}
\end{aligned}
$$

where Property 2.2 has been used. For simplicity, set $\Lambda_{1}$ $=\Lambda_{2}=\Lambda$ and $K_{p}=L_{p \mid}$. So,

$$
\begin{gathered}
-e^{T} \Lambda_{1} K_{p} e+\tilde{q}^{T} \Lambda_{1} K_{p} e-\tilde{q}^{T} \Lambda_{2} L_{p 1} \tilde{q} \\
=-\frac{1}{2} e^{T} \Lambda K_{p} e-\frac{1}{2} \tilde{q}^{T} \Lambda K_{p} \tilde{q} .
\end{gathered}
$$

Let us replace Assumption 2.3 by Assumption 2.4

Assumption 2.4: The desired trajectory velocity $\dot{q}_{d}$ is bounded by $V_{M}$, i.e.,

$$
V_{M}=\sup _{t}\left\|\dot{q}_{d}(t)\right\| .
$$

Next, using Property 2.3 and completion of the squares, (61) can be upper bounded by

$$
\begin{aligned}
\dot{H}_{3}\left(e, s_{1}, \tilde{q}, s_{2}\right) & \\
\leq & -\left(K_{d, m}-C_{M}\left(V_{M}+\|\Lambda e\|+\Lambda \tilde{q} \|\right.\right. \\
& \left.\left.+\left\|s_{2}\right\|\right)\right)\left\|s_{1}\right\|^{2}-\left(l_{d} M_{m}-K_{d, M}\right. \\
& \left.-C_{M}\left(V_{M}+\|\Lambda e\|+\|\Lambda \tilde{q}\|+\left\|s_{1}\right\|\right)\right) \\
& \cdot\left\|s_{2}\right\|^{2}-\frac{1}{2} K_{p, m} \Lambda_{M}^{-1}\|\Lambda \tilde{q}\|^{2} \\
& -\frac{1}{2} K_{p, m} \Lambda_{M}^{-1}\|\Lambda e\|^{2}
\end{aligned}
$$

Then the following proposition is obtained.

Proposition 3.1: Consider the passivity-based outputfeedback robot tracking controller

Controller $\left\{\begin{aligned} \tau= & M(q) \ddot{q}_{r}+C\left(q, \dot{q}_{0}\right) \dot{q}_{r}+G(q) \\ & -K_{d}\left(\dot{q}_{0}-\dot{q}_{r}\right)-K_{p} e \\ \dot{q}_{r}= & \dot{q}_{d}-\Lambda\left(\hat{q}-q_{d}\right) \\ \dot{q}_{0}= & \dot{\hat{q}}-\Lambda(q-\hat{q})\end{aligned}\right.$

Observer $\left\{\begin{aligned} \dot{\hat{q}}= & z+L_{d}(q-\hat{q}) \\ \dot{z}= & \ddot{q}_{r}+L_{p 2}(q-\hat{q}) \\ & +M^{-1}(q) K_{p}\left(q_{d}-\hat{q}\right)\end{aligned}\right.$ 
where $L_{d}$ and $L_{p 2}$ satisfy Assumption 2.2. Under the following conditions:

$$
\begin{aligned}
K_{d, m} & >C_{M} V_{M} \\
l_{d} & >M_{m}^{-1}\left(K_{d, M}+C_{M} V_{M}\right)
\end{aligned}
$$

the closed-loop system is locally exponentially stable. Moreover, a region of attraction is given by

$$
B=\left\{x \in \mathbb{4}^{4 n} \mid\|x\|<\frac{1}{1+\sqrt{2}} \delta \sqrt{\frac{P_{m}}{P_{M}}}\right\}
$$

where $x^{T}=\left[s_{1}^{T}(\Lambda e)^{T} s_{2}^{T}(\Lambda \tilde{q})^{T}\right]$,

$$
\delta=\min \left\{\frac{l_{d} M_{m}-K_{d, M}}{C_{M}}-V_{M}, \frac{K_{d, m}}{C_{M}}-V_{M}\right\}
$$

and $P_{m}, P_{M}$ are defined as

$$
\begin{aligned}
& P_{m}=\min \left\{M_{m}, \Lambda_{M}^{-2} K_{p, m}\right\}, \\
& P_{M}=\max \left\{M_{M}, \Lambda_{m}^{-2} K_{p, M}\right\} .
\end{aligned}
$$

Consider also the following Corollary.

Corollary 3.1: For position control, i.e., $q_{d}=$ constant, under the condition

$$
l_{d}>M_{m}^{-1} K_{d, M}
$$

the tracking controller (65) is locally exponentially stabilizing.

Proof of Proposition 3.1: Take as a Lyapunov function $V(x)=H_{3}\left(e, s_{1}, \tilde{q}, s_{2}\right)$, where $H_{3}\left(e, s_{1}, \tilde{q}, s_{2}\right)$ as defined in (60). This function satisfies

$$
\frac{1}{2} P_{m}\|x\|^{2} \leq V(x) \leq \frac{1}{2} P_{M}\|x\|^{2}
$$

where $P_{m}, P_{M}$ as defined in (69). Then the proof follows along the same lines as the proof of Proposition 2.1.

From (67-69) it can be verified that the attraction region grows either $O\left(l_{d}\right)$ or $O\left(K_{d}\right)$, depending on $\delta$ in (68), which implies that the result is semiglobal.

\section{B. Discussion}

1) In a separate controller and observer design approach the controller (15) would have been combined with the open-loop observer (23). In that case the observer system would have obtained the following structure:

$$
\text { Observer }\left\{\begin{aligned}
& \dot{\hat{q}}=z+L_{d} \tilde{q} \\
& \dot{z}= \ddot{q}_{r}+L_{p 2} \tilde{q}+M^{-1}(q)\left[K_{p}\left(q_{d}-\hat{q}\right)\right. \\
&\left.+C\left(q, \dot{q}_{0}\right)\left(\dot{q}_{r}-\dot{q}_{0}\right)-K_{d}\left(\dot{q}_{r}-\dot{q}_{0}\right)\right] .
\end{aligned}\right.
$$

The actual observer (65b) has a simpler structure. First, the compensation term $C\left(q, \dot{q}_{0}\right)\left(\dot{q}_{r}-\dot{q}_{0}\right)$ has been removed. Second, there is no need to feed forward the part $-K_{d}\left(\dot{q}_{r}-\dot{q}_{0}\right)$ of the control input into the observer system, since in the time derivative (61) of the desired energy function the cross term $s_{1}^{T} K_{d} s_{2}$, which is due to the right-hand side of (56), is canceled by the cross term -
$s_{2}^{T} K_{d} s_{1}$, which comes from the right-hand side of (59). In particular this feature illustrates the advantage that is obtained by an efficient tuning of the controller and observer structure to each other.

2) The "filtered error signals" $s_{1}, s_{2}$, as defined in (51) and (54), respectively, could have been chosen differently. For example, by defining

$$
s_{2}=\dot{\tilde{q}}+\Lambda_{2} \tilde{q}+\Lambda_{2} e
$$

one can increase the design freedom. Also the generalizations as discussed in Remark 2.2 can be employed.

3) The choices $\dot{q}_{0}$ and $\dot{q}_{r}$ in the compensation for the Coriolis and centrifugal torque, see (65), are in some sense arbitrary. In particular, it can be shown that for any compensation of the form $C\left(q, \dot{q}_{c, 1}\right) \dot{q}_{c, 2}$, where $\dot{q}_{c, 1}, \dot{q}_{c, 2}$ consists of some combination of $\dot{q}_{d}, \dot{\hat{q}}, \Lambda_{1} e$, and $\Lambda_{2} \tilde{q}$, semiglobal exponential stability can be warranted under similar conditions on the controller and observer gains. Therefore actually a whole family of exponentially stabilizing output-feedback tracking controllers has been developed (cf. [22]).

4) Passivity interpretation-it is straightforward to interpret the controller-observer combination in terms of passivity. To that end, rewrite the error dynamics (56) and (59) as follows (take for simplicity $K_{p}=L_{p 1}=0$ )

$$
\begin{aligned}
\bar{M}(q) \dot{s}+\bar{C}(q, \dot{q}) s & =\nu \\
\nu & =-T(q, \dot{q}, s) s
\end{aligned}
$$

where

$$
\begin{aligned}
s^{T} & =\left[\begin{array}{ll}
s_{1}^{T} & s_{2}^{T}
\end{array}\right] \\
\bar{M}(q) & =\operatorname{diag}[M(q), M(q)] \\
\bar{C}(q, \dot{q}) & =\operatorname{diag}[C(q, \dot{q}), C(q, \dot{q})]
\end{aligned}
$$

$T(q, \dot{q}, s)$

$$
=\left[\begin{array}{cc}
K_{d} & -\left[K_{d}+C\left(q, s_{1}-\dot{q}\right)\right] \\
K_{d}-C\left(q, s_{2}-\dot{q}\right) & l_{d} M(q)-K_{d}
\end{array}\right] .
$$

It can easily be shown that the mapping from $\nu$ to $s$ in (73a) is passive. The block (73b) is strictly passive from $s$ to $-\nu$ if $T(x)>0$, which is satisfied under the assumptions of Proposition 3.1.

\section{The Paden and Panja Scheme for Output Feedback}

The motion controller (15) is very elegant in the sense that it optimally shapes the closed-loop dynamics in order to meet the desired energy function (18). Unfortunately, this controller does not satisfy the reduction principle in case of regulation, unlike the passivity-based controller (13) of Paden and Panja [6]. For this reason it is interesting to modify (13) to an output-feedback system. This is considered in the following.

Step 1: Choose $s_{1}, s_{2}$ as in (17), (28), respectively, and take (49) as desired energy function, where

$K_{1}(q, e)=\Lambda_{1}\left[2 K_{d} \Lambda_{1}^{-1}-M(q)\right] \Lambda_{1}, K_{2}(q, \tilde{q})=2 \Lambda_{2} K_{d}$. 
Define, based on (49)-(74), the passivity-based outputfeedback robot controller

$$
\begin{gathered}
\text { Controller }\left\{\begin{array}{c}
\tau=M(q) \ddot{q}_{d}+C\left(q, \dot{q}_{0}\right) \dot{q}_{d} \\
+G(q)-K_{d}\left(\dot{q}_{0}-\dot{q}_{r}\right)
\end{array}\right. \\
\text { Observer }\left\{\begin{array}{l}
\dot{q}=z+L_{d} \tilde{q} \\
\dot{q}_{r}=\dot{q}_{d}-\Lambda_{1} e \\
\dot{\dot{q}}=\ddot{q}_{d}+L_{p 2} \tilde{q} .
\end{array}\right.
\end{gathered}
$$

Step 2: Via straightforward calculation it can be shown that the time-derivative of the desired energy function (49), (74) along the error dynamics (1), (75) is given by

$$
\begin{aligned}
\dot{H}_{3}(x)= & -x^{T} Q(x) x-s_{2}^{T}\left(l_{d} M(q)-2 K_{d}\right) s_{2} \\
& -s_{1}^{T} C\left(q, s_{2}\right) \dot{q}_{d}+\dot{e}^{T} C(q, \dot{q})\left(\Lambda_{1} e\right) \\
& +s_{2}^{T} C\left(q, s_{2}-\dot{q}\right) \dot{e}
\end{aligned}
$$

with

$$
Q(x)=\left[\begin{array}{cc}
{\left[\begin{array}{cc}
K_{d}-\Lambda_{1} M(q) & 0 \\
0 & K_{d}
\end{array}\right]} & 0 \\
0 & {\left[\begin{array}{cc}
K_{d} & 0 \\
0 & K_{d}
\end{array}\right]}
\end{array}\right]
$$

where $x(t)^{T}=\left[\dot{e}(t)^{T}\left[\Lambda_{1} e(t)\right]^{T} \dot{\tilde{q}}(t)^{T}\left[\Lambda_{2} \tilde{q}(t)\right]^{T}\right]$. Then we have the following proposition:

Proposition 3.2: Consider (75), where $L_{d}$ and $L_{p 2}$ satisfy Assumption 2.2. Under the following conditions:

$$
\begin{aligned}
K_{d, m} & >\Lambda_{1, M} M_{M}+(3+\sqrt{2}) C_{M} V_{M} \\
l_{d} & >2 M_{m}^{-1} K_{d, M}
\end{aligned}
$$

the closed-loop system is locally exponentially stable. A region of attraction is given by

$$
\begin{aligned}
B= & \left\{x \in 4 n \mid\|x\|<\delta \sqrt{\frac{P_{m}}{P_{M}}}\right. \\
& \left.\cdot\left[\frac{\left(K_{d}-\Lambda_{1, M} M_{M}\right)}{C_{M}}-(3+\sqrt{2}) V_{M}\right]\right\}
\end{aligned}
$$

where $\delta=(1 /(3+\sqrt{2}))$, and

$$
\begin{aligned}
& P_{m}=\min \left\{\frac{1}{3} M_{m}, \frac{2}{3} \Lambda_{2, M}^{-1} \Lambda_{1, M} M_{M}\right\}, \\
& P_{M}=\max \left\{6 \Lambda_{1, m}^{-1} K_{d, M}, 6 \Lambda_{2, m}^{-1} K_{d, M}\right\}
\end{aligned}
$$

Proof: Analog to the proof of Proposition 3.1. For further details, see [27].

\section{1) Discussion}

1) The result in this section was obtained via an optimal tuning of the controller and observer system. In particular, the controller consists of a linear estimated state- feedback part and a nonlinear part that compensates for the robot dynamics. The estimated velocity is provided by a second-order linear observer, into which the desired acceleration is fed forward.

2) It can easily be verified that the closed-loop error dynamics (1)-(75) are also characterized by passivity preservation (cf. point 4 ) in the discussion of Section III-B.

3) In the case of position control, the following modified controller version of (8)-(12) is obtained (for $\Lambda_{1}=$ $\Lambda_{2} \equiv \Lambda$ and $K_{p} \equiv K_{d} \Lambda$ )

$$
\begin{aligned}
& \text { Controller }\left\{\tau=G(q)-K_{d} \dot{\hat{q}}-K_{p}\left(\hat{q}-q_{d}\right)\right. \\
& \text { Observer }\left\{\begin{array}{l}
\dot{\hat{q}}=z+L_{d}(q-\hat{q}) \\
\dot{z}=L_{p 2}(q-\hat{q}) .
\end{array}\right.
\end{aligned}
$$

Equation (81) underscores the simplicity of the controller-observer combination (75).

4) The controller-observer combination (75) is based on the requirement that exact knowledge of the robot dynamics is available. Obviously, this is a rather strong requirement that generally cannot be met in practice. In this respect it is interesting to mention that in a recent result the system (1)-(75) was shown to be semiglobally uniformly ultimately bounded (SUUB) in the presence of arbitrary uncertainties in the rigid robot dynamics $M(q)$, $C(q, \dot{q})$, and $G(q)$; see [28]. Moreover, this SUUB result remains valid under unknown friction and load disturbances. This indicates that the proposed passivity-based output-feedback controllers preserve the inherent robustness properties of passivity-based state-feedback systems!

5) For practical implementation it is also important to analyze the noise robustness of the control system (75). To this end, assume that the position measurements $q$ are contaminated with noise $\eta$. Then, (75b) becomes

$$
\dot{\hat{q}}=z+L_{d}(q+\eta-\hat{q}) \text {. }
$$

From (82) it is easy to see that the velocity estimate $\dot{\hat{q}}$ is quite sensitive for noise on position measurements. This is undesirable since it may limit the choice of the derivative feedback gain $K_{d}$ and, therefore, the closed-loop bandwidth.

To diminish the noise sensitivity problem, it may be advantageous to replace the controller part $\dot{\hat{q}}$ by the integrator output $z$, see (75b), which is more immune to noise. So assume that (75a) is redefined as

$$
\text { Controller } \begin{aligned}
\{\tau= & M(q) \ddot{q}_{d}+C\left(q, \dot{q}_{0}\right) \dot{q}_{d}+G(q) \\
& -K_{d}\left(z-\dot{q}_{r}\right)
\end{aligned}
$$

where $\dot{q}_{0}, \dot{q}_{r}$ as in (75a). The closed-loop system (1)-(83), (75b) can be shown to be locally exponentially stable under conditions on the controller and observer gains that are slightly stronger than (78) [27]. The proof basically follows along the same lines as the one of Proposition 3.2. Recent experimental results [27] demonstrate, however, that in practice the noise rejection features of $(83)$ are not better compared to those of $(75)$, since for stable 
control performance the observer bandwidths need to be selected equivalent for both (75) and (83).

6) In practical robot control proportional-derivative feedback generally constitutes the main loop, because of its robustness, reliability, and acceptable performance. The passivity concept actually theoretically justifies the usefulness of PD feedback by showing that it exploits the physical nature of the robot system. If velocity information is noisy or even not present, a practically feasible solution is to implement the derivative part by reconstructing the velocity signal via linear estimation techniques, particularly because of its ease of implementation. The results of this section form a scientific basis for this approach.

\section{Experimental Case Study}

To verify the theoretical analysis, a series of experiments were performed with the controller (75) using a two-DOF robot manipulator moving in the vertical plane; see Fig. 1 [29]. This robot system is actually lightweight in construction, containing some flexibility in the links. Nevertheless, under relatively slow motion assumptions the system matches the rigid body dynamics described in the Appendix. For these dynamics it can be shown that $M_{m}=1 \mathrm{~kg} \cdot \mathrm{m}^{2}, M_{M}=25 \mathrm{~kg} \cdot \mathrm{m}^{2}$, and $C_{M}=6 \mathrm{~kg} \cdot \mathrm{m}^{2}$ $\cdot \mathrm{s}^{-1}$.

Controller Implementation Issues: The robot is controlled using a network of transputers, which offers a powerful tool for real-time control. This network consists of a data acquisition rack based on the Twente LINX backplane [30] and a MEIKO computing surface, which are connected by means of an optical transputer link. The data acquisition and actuation takes place using two 16bit T222 transputers, one for each robot link. The transputers inside the MEIKO cabinet perform the control calculations on 32-bit T800 transputers. The control algorithm was distributed on two T800's, one containing the velocity observer and the PD-feedback, and the other containing the model-based components. This separation on two transputers was performed to facilitate the use of dual rate control, which allows to update the model-based portion at a slower rate than the PD-loop including observer. See, for instance [31], where a similar idea was used. With this parallel processing equipment sampling frequencies of up to $3 \mathrm{kHz}$ can be obtained, although the actual sampling frequency was set at $1 \mathrm{kHz}$ for the PD and observer part, and $250 \mathrm{~Hz}$ for the model-based part. Position measurements are obtained from resolvers that are mounted directly on the motor shafts. To convert the analog resolver signals, 16 bits RDC's are used. Actuation takes place using a 12 bit D/A converter. Further details can be found in the work of Roebbers [30]

We utilized as much as possible the knowledge of the system dynamics in the controller implementation. For that reason, the model-based part in the controller (75) was extended with an explicit compensation for the friction $F(\dot{q})$ present in the system. This friction can approx-

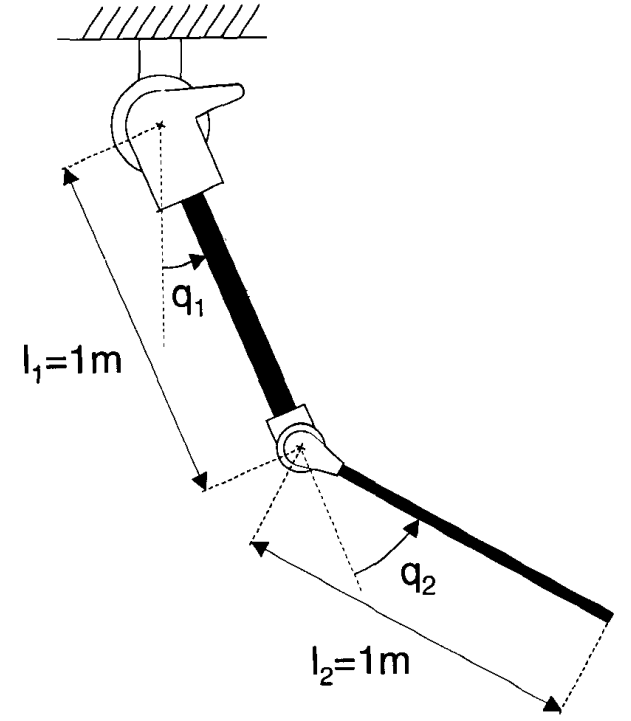

Fig. 1. Two-DOF robot system.

imately be described by Coulomb and viscous terms [27], as can be seen from the structure of $F(\cdot)$ given in the Appendix. The friction compensation strategy is based on the desired trajectory velocity, in order to prevent chattering of the sign-dependent terms at low velocities. According to the foregoing, the actual control input was given by

$$
\text { Controller } \begin{aligned}
\tau= & M(q) \ddot{q}_{d}+C\left(q, \dot{q}_{0}\right) \dot{q}_{d}+G(q) \\
& +F\left(\dot{q}_{d}\right)-K_{d}\left(\dot{q}_{0}-\dot{q}_{r}\right)
\end{aligned}
$$

where $\dot{q}_{r}$ and $\dot{q}_{0}$ as in (75a), and the observer part as given in $(75 \mathrm{~b})$. The integrators in he observer dynamics were implemented using the forward Euler approximation, which shows that the observed part is computationally simple.

Reference Trajectory: In these experiments the robot system had to follow a desired path given in Fig. 2. An upper bound on the velocity of this path, see Assumption 2.4 , is given by $V_{M}=0.4 \mathrm{rad} \cdot \mathrm{s}^{-1}$. As mentioned above, the desired path is selected relatively slow in order to prevent strong excitation of the unmodeled flexibility effects in the links.

Results Using Controller-Observer Combination: In a first experiment we considered the closed-loop performance of $(75 b)-(84)$, with the controller and observer gains equal to

$$
\begin{aligned}
& K_{d}=\left[\begin{array}{rr}
100 & 0 \\
0 & 30
\end{array}\right], \quad \Lambda_{1}=\left[\begin{array}{rr}
15 & 0 \\
0 & 10
\end{array}\right], \quad l_{d}=250, \\
& \Lambda_{2}=\left[\begin{array}{rr}
0.1 & 0 \\
0 & 0.1
\end{array}\right] .
\end{aligned}
$$



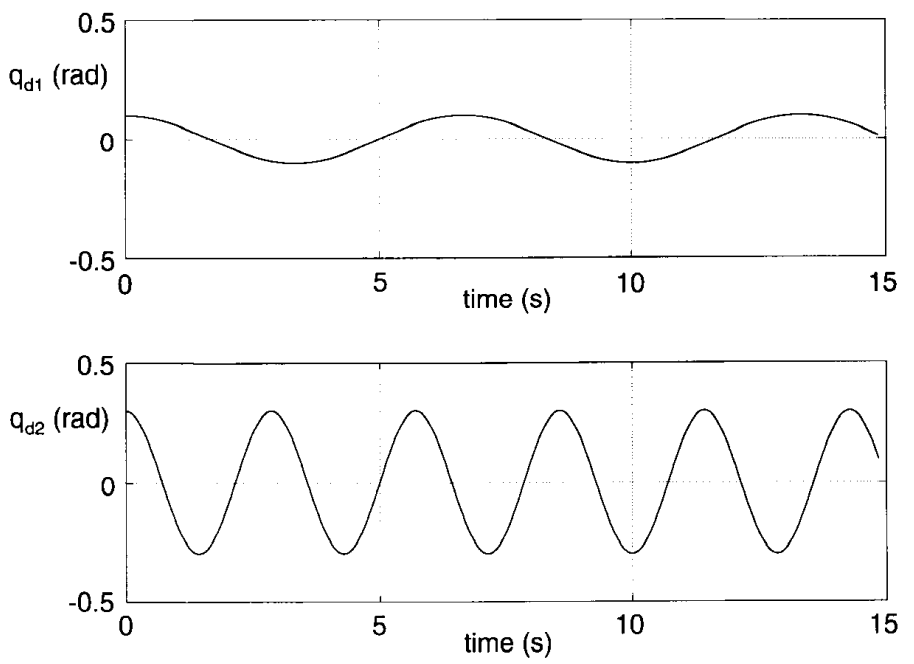

Fig. 2. Desired trajectory signals.

The initial observer conditions were set at

$$
\hat{q}(0)=q(0), z(0)=\left[\begin{array}{l}
0 \\
0
\end{array}\right]
$$

which corresponds to the fact that position measurements are available. In Fig. 3 the tracking errors $e(t)$ are given, for both the upper (a) and lower (b) link. For clarity the errors are shown on large and small scales. Fig. 4 shows the corresponding velocity error signal, i.e., $\dot{q}_{0}(t)-\dot{q}_{d}(t)$.

From Fig. 3 it can be concluded that the transient responses are slightly underdamped, which is due to the relatively large proportional action $\Lambda_{1}$. Unfortunately, decreasing $\Lambda_{1}$ would yield a smaller loop stiffness, which in turn would decrease the tracking accuracy after the transient phase. Since we prefer a high tracking accuracy, $\Lambda_{1}$ was chosen to be large, at the cost of underdamped initial responses.

Results Using Numerical Differentiation of Position:

Next we performed a comparative experiment in which the velocity was approximated by the in practice frequently employed numerical position differentiation technique. That is, we defined the velocity estimate in the control input (84) as

$$
\dot{q}_{0}(k T)=\frac{q(k T)-q((k-1) T)}{T}, \quad k=1, \cdots, d
$$

where $k$ represents the sampling instant, $T=1 \mathrm{~ms}$, and $d T=15 \mathrm{~s}$, the total experiment time. The results of this experiment are presented in Figs. 5 and 6.

Figs. 3 and 5 illustrate that the tracking errors for both velocity reconstruction approaches are similar in both the transient and "steady-state" phase. On the other hand, the velocity errors in Figs. 4 and 6 show a clear differ- ence: the second-order linear observer (75b) provides a velocity estimate that has a significant higher immunity to noise compared to the one of the differentiation technique (87). This is also indicated by the variance of the velocity error signals, which are enumerated in Table I. These variances have been determined on $t \in[2.5,15]$ using the MATLAB-function $\operatorname{cov}(\cdot)$.

Table I shows that the variance has at least been decreased a factor four. This is attractive for two reasons. First, it yields a smoother control input, which may both increase the actuator's lifetime and prevent the excitation of unmodeled high-frequency dynamics. Second, it allows increase of the derivative gain $K_{d}$ and therefore the proportional gain $\Lambda_{1}$, which enables the achievement of higher levels of tracking performance (cf. [28]).

Two aspects need further discussion. First, despite the different noise levels in the velocity estimate and consequently the input torque, the tracking errors show basically the same time behavior. This can be explained from the second-order filtering nature of the robot dynamics from torque to position. Second, not all of the 16 bits of the lower link RDC are significant. In particular, the accuracy of the position signal of the upper link is 16 bits, whereas that of the lower link is only 14 bits due to crosstalk effects in the data acquisition rack. This point can clearly be observed from Fig. 6: the noise on the estimated velocity of the upper link is mainly determined by quantization noise, whereas the noise on the velocity estimate of the lower link is dominated by electromagnetic disturbances.

Remark 4.1: Although the controller-observer system shows acceptable tracking performance, the choice (85) for $K_{d}$ does not satisfy the condition (78a) as required by Proposition 3.2 for asymptotic stabilization. This indicates that the conditions on the gains as given in the Propositions $2.1,3.1$, and 3.2 are likely to be conservative, and will in general not be necessary. 

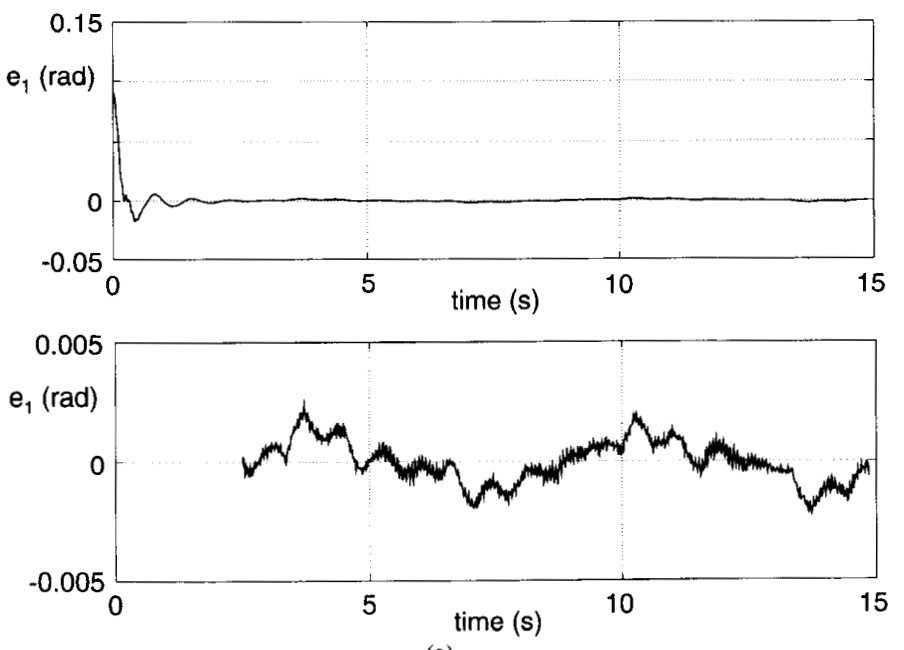

(a)
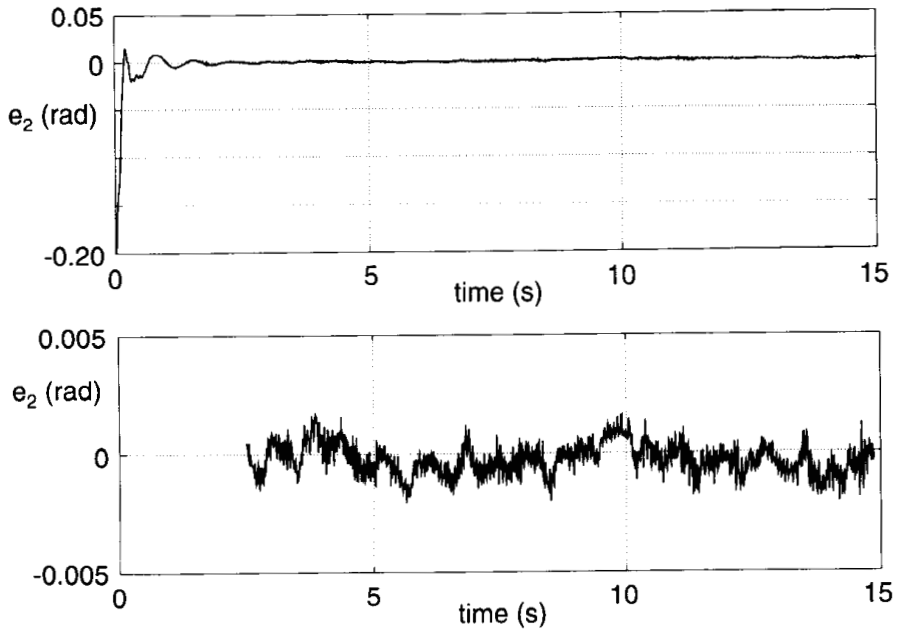

(b)

Fig. 3. Tracking errors for controller-observer system (4.1)-(3.26b). (a) Upper link. (b) Lower link.
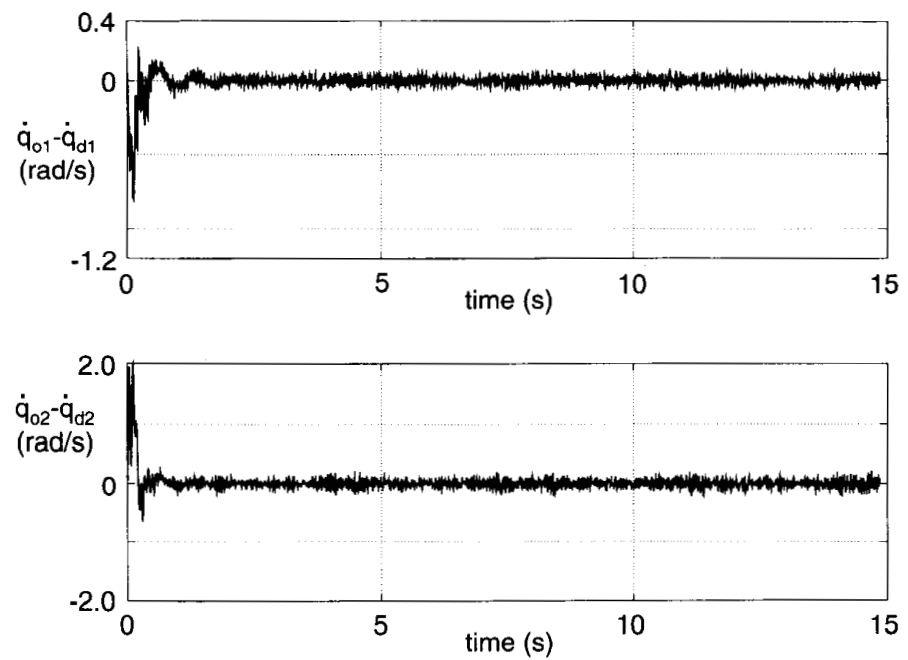

Fig. 4. Velocity errors for (4.1)-(3.26b). 

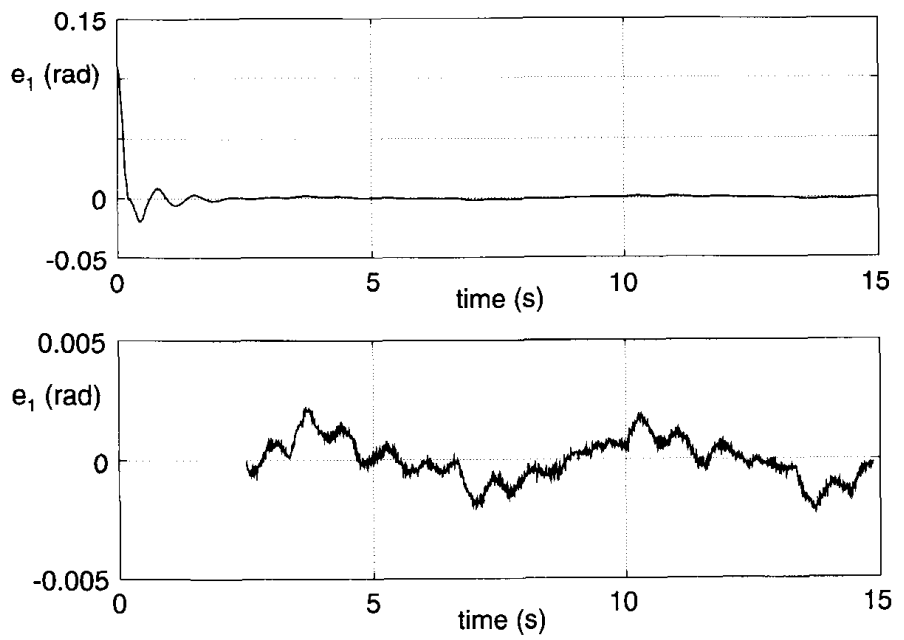

(a)
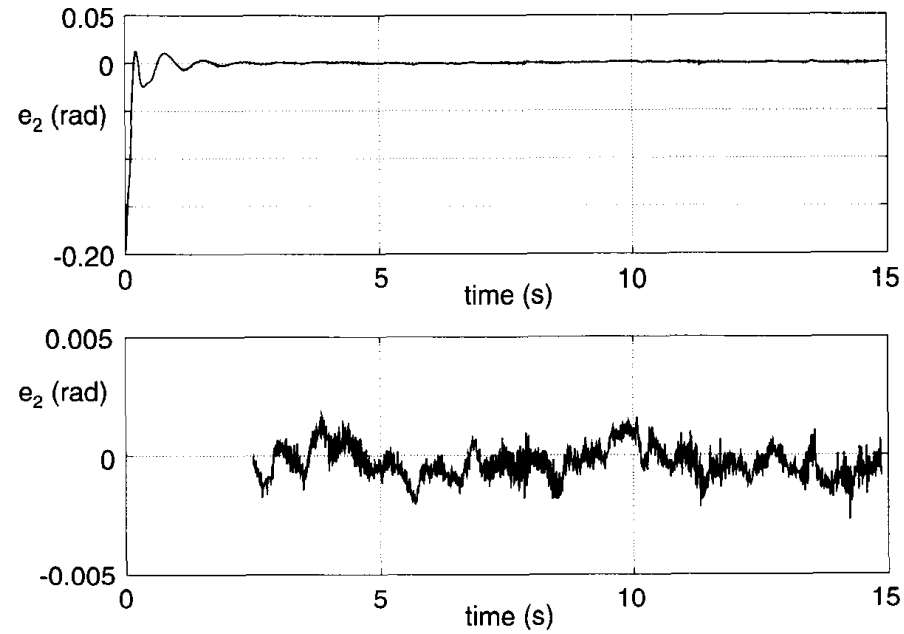

(b)

Fig. 5. Tracking errors for controller-observer system (4.1), (4.4). (a) Upper link. (b) Lower link.
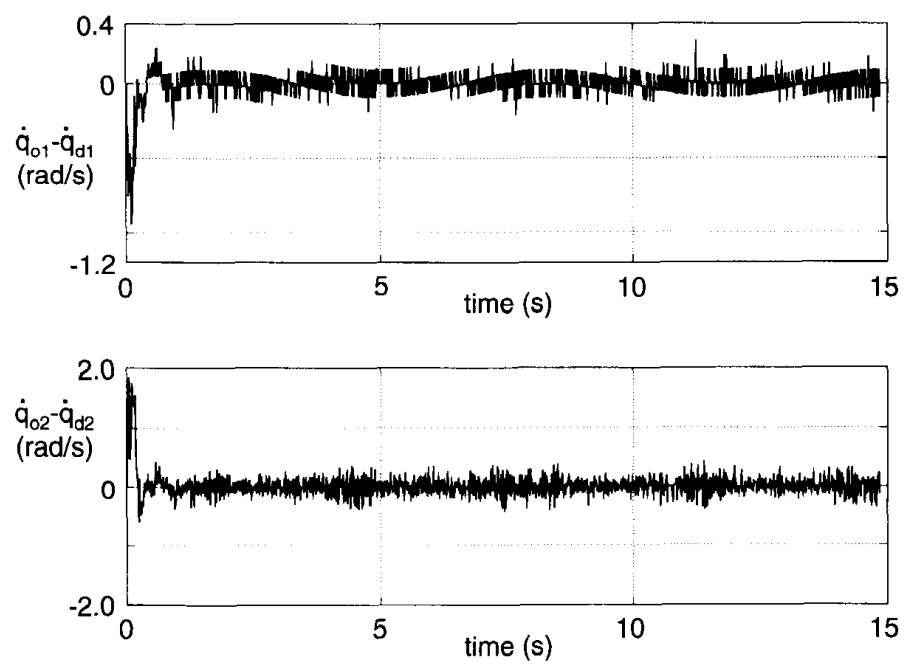

Fig. 6. Velocity errors for (4.1), (4.4) 
TABLE I

VARIANCES $\left[\operatorname{RAD}^{2} \cdot \mathrm{s}^{-2}\right]$ OF VELOCITY ERROR SIGNALS

\begin{tabular}{|l|c|c|}
\hline Variance & Observer (3.26b) & Differentiation (4.4) \\
\hline Upper link & $4.810^{-4}$ & $3.910^{-3}$ \\
\hline Lower link & $4.310^{-3}$ & $1.810^{-2}$ \\
\hline
\end{tabular}

\section{CONCLUSION}

A strategy for designing robot motion control systems that requires only joint position measurements was presented. To this end the passivity approach to state-feedback robot control was extended in a natural way to the case that a velocity observer is integrated in the control loop. Via some illustrative design examples it was shown that this strategy can provide semiglobally exponentially stabilizing solutions to the output-feedback robot tracking problem that are computationally efficient and robust to model uncertainties, which is mainly due to the fact that in the resulting control systems both the controller and observer exploit the physical structure of the robot system.

Tests on a two-DOF mechanical construction were performed in order to experimentally verify the characteristic features of, from an implementation perspective, the most interesting controller-observer combination. It was illustrated that the tracking errors converge (close) to zero, although the conditions on the controller gains for stability were not met. This indicates that these stability conditions are too conservative and in general not necessary. More interestingly, comparative experiments showed that the linear observer outperforms the rather ad hoc position differentiation algorithm, with respect to noise sensitivity. This is achieved despite the fact that the additional computations for the linear observer are basically negligible.

We would like to stress that the presented control schemes, particularly the one proposed in Section III-B, are interesting for industrial purposes. As is well known, an important design constraint in the development of robotic and other mechatronic systems is their economic feasibility. Because of the considerable cost savings that can be obtained, many robots today determine a velocity from the position signal by a first-order approximation [12]. The velocity observer as presented in Section III-B may serve as a useful alternative for such an approximation, particularly because of the guaranteed stability and enhanced noise-rejection properties.

\section{APPENDIX}

The robot system is characterized by [27]

$$
M(q)=\left[\begin{array}{cc}
9.77+2.02 \cos \left(q_{2}\right) & 1.26+1.01 \cos \left(q_{2}\right) \\
1.26+1.01 \cos \left(q_{2}\right) & 1.12
\end{array}\right]
$$

$$
\begin{gathered}
C(q, \dot{q})=\left[\begin{array}{cc}
-1.01 \sin \left(q_{2}\right) \dot{q}_{2} & -1.01 \sin \left(q_{2}\right)\left(\dot{q}_{1}+\dot{q}_{2}\right) \\
1.01 \sin \left(q_{2}\right) \dot{q}_{1} & 0
\end{array}\right] \\
G(q)=g\left[\begin{array}{c}
8.1 \sin \left(q_{1}\right)+1.13 \sin \left(q_{1}+q_{2}\right) \\
1.13 \sin \left(q_{1}+q_{2}\right)
\end{array}\right] \\
F(\dot{q})=\left[\begin{array}{l}
\dot{q}_{1}+3 \operatorname{sign}\left(\dot{q}_{1}\right) \\
1.2 \dot{q}_{2}+\operatorname{sign}(\dot{q})
\end{array}\right]
\end{gathered}
$$

where $g=9.8 \mathrm{~ms}^{-2}$ is the acceleration of gravity.

\section{REFERENCES}

[1] J. Y. S. Luh, M. W. Walker, and R. P. C. Paul, "Resolved acceleration control of mechanical manipulators," IEEE Trans. Automat. Contr., vol. 25, pp. 468-474, 1980.

[2] H. Nijmeijer and A. J. van der Schaft, Nonlinear Dynamical Control Systems, Berlin: Springer-Verlag, 1990.

[3] R. Ortega and M. W. Spong, "Adaptive motion control of rigid robots: A tutorial," Automatica., vol. 25, pp. 877-888, 1989.

[4] A. J. van der Schaft, "System theory and mechanics in three decades of mathematical system theory," in Lecture Notes in Control and Information Sciences, H. Nijmeijer and J. M. Schumacher, Eds. Berlin: Springer-Verlag, 1990 , pp. 425-452.

[5] M. Takegaki and S. Arimoto, "A new feedback method for dynamic control of manipulators," ASME J. Dynam. Syst., Measurement, Control, vol. 102, pp. 119-125, 1981.

[6] B. Paden and R. Panja, "Globally asymptotically stable 'PD +' controller for robot manipulators,"' Int. J. Control, vol. 47, pp. 1697$1712,1988$.

[7] R. Kelly, R. Carelli, and R. Ortega, "Adaptive motion control design of robot manipulators: An input-output approach," Int. J. Control, vol. 50, pp. 2563-2581, 1989.

[8] J. J.-E. Slotine and W. Li, "On the adaptive control of robot manipulators," Int. J. Robotics Res., vol. 6, pp. 49-59, 1987.

[9] I. D. Landau and R. Horowitz, "Applications of the passive systems approach to the stability analysis of adaptive controllers for robot manipulators," Int. J. Adaptive Control and Signal Processing, vol. 3, pp. 23-38, 1989.

[10] P. K. Khosla and T. Kanade, "Experimental evaluation of nonlinear feedback and feedforward control schemes for manipulators," Int. J. Robotics Res., vol. 7, pp. 18-28, 1988.

[11] R. Gourdeau and H. M. Schwartz, "Adaptive Control of Robotic Manipulators: Experimental Results," in Proc. IEEE Conf. Robotics Automat., vol. 1, Sacramento, CA, Apr. 1991, pp. 8-15.

[12] R. D. Klafter, T. A. Chmielewski, and M. Negin, Robotic Engineering-An Integrated Approach. Englewood Cliffs, NJ: PrenticeHall, 1989.

[13] P. R. Bélanger, "Estimation of angular velocity and acceleration from shaft encoder measurements," in Proc. IEEE Conf. Robotics Automat., Nice, France, May 1992, pp. 585-592.

[14] C. Canudas de Wit, and N. Fixot, "Robot control via robust state estimated feedback," IEEE Trans. Automat. Contr., vol. 36, pp. 1497-1501, 1991.

[15] C. Canudas de Wit and J.J.-E. Slotine, "Sliding observers for robot manipulators," Automatica, vol. 27, pp. 859-864, 1991.

[16] J. J.-E. Slotine, J. K. Hedrick, and E. A. Misawa, "Sliding observers for nonlinear systems," ASME J. Dynam. Syst., Measurement, Control, vol. 109, pp. 245-252, 1987.

[17] S. Nicosia, A. Tomambé, and P. Valigi, "Experimental results in state estimation of industrial robots," in Proc. Conf. Decision Control, Honolulu, HI, Dec. 1990 , pp. $360-365$

[18] C. Canudas de Wit, N. Fixot, and K. J. Å ström, "Trajectory tracking in robot manipulators via nonlinear estimated state feedback," IEEE Trans. Robotics Automat., vol. 8, pp. 138-144, 1992.

[19] S. Nicosia and P. Tomei, "Robot control by using only joint position measurements," IEEE Trans. Automat. Contr., vol. 35, pp. 1058 1061,1990 
[20] H. Berghuis, H. Nijmeijer, and P. Löhnberg, "Observer design in the tracking control problem of robots," in Proc. IFAC Symp. NOLCOS'92, Bordeaux, France, June 1992, pp. 588-593.

[21] N. Sadegh and R. Horowitz, "Stability and robustness analysis of a class of adaptive controllers for robotic manipulators," Int. J. Robotics Res., vol. 9, pp. 74-92, 1990.

[22] J. T. Wen and D. S. Bayard, "New class of control laws for robotic manipulators: Part 1. Non-adaptive Case,", Int. J. Control., vol. 47, pp. 1361-1385, 1988.

[23] L. L. Whitcomb, A. A. Rizzi, and D. E. Koditschek, "Comparative experiments with a new adaptive controller for robot arms," IEEE Trans. Robotics Automat., vol. 9, pp. 59-70, 1993.

[24] C. A. Desoer and M. Vidyasagar, Feedback Systems: Input-Output Properties. New York: Academic, 1975.

[25] H. Kwakernaak and R. Sivan, Linear Optimal Control Systems. New York: Wiley, 1972.

[26] R. Ortega and G. Espinosa, "A controller design methodology for systems with physical structures: Application to induction motors," in Proc. IEEE Conf. Decision Control, vol. 3, Brighton, England, Dec. 1991, pp. 2345-2349.

[27] H. Berghuis, "Model-based robot control: From theory to practice," Ph.D. dissertation, Univ. Twente, Enschede, The Netherlands, 1993.

[28] H. Berghuis and H. Nijmeijer, "Robust control of robots using only position measurements," in Proc. IFAC World Congress, Sydney, Australia, July 1993, vol. 1, pp. 501-506.

[29] L. Kruise, "Modelling and control of a flexible beam and robot arm," Ph.D. dissertation, Univ. Twente, Enschede, The Netherlands, 1990.

[30] H. Roebbers, "Detailed report on the realization of STW-project TEL 70.1389," Intern. Rep. 92R197, Dept. Electr. Eng., Univ. Twente, Enschede, The Netherlands.

[31] N. Sadegh and K. Guglielmo, "Design and implementation of adaptive and repetitive controllers for mechanical manipulators," IEEE Trans. Robotics Automat., vol. 8, pp. 395-400, 1992.

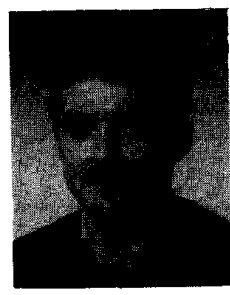

Harry Berghuis (S'93) was born on September 15,1965 , in Westerhaar, The Netherlands. In 1988 he received the M.Sc. degree (cum laude) in electrical engineering from the University of Twente, Enschede, The Netherlands.

Subsequently, he started his $\mathrm{Ph} . \mathrm{D}$. research on model-based robot control at the same university, on a joint project between the Systems and Control Group at the Department of Applied Mathematics and the Control Group at the Department of Electrical Engineering. This project was completed in 1993. His current research interests include nonlinear control applications, control of robots in dynamic interaction with the environment, and mobile robot control.

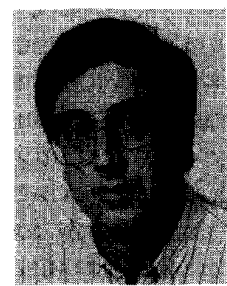

Henk Nijmeijer (SM'93) received both his university degree and doctorate in mathematics from the University of Groningen, Groningen, The Netherlands, in 1979 and 1983, respectively.

After spending three years at the Center for Mathematics and Computer Science (CWI), Amsterdam, The Netherlands, he joined the Department of Applied Mathematics, Twente University of Technology, Enschede, The Netherlands in 1983, where he is presently an Associate Professor (UHD). His main research interests lie in the area of nonlinear and linear geometric control theory and its applications. He is an author (with A. J. Van der Schaft) of Nonlinear Dynamical Control Systems (Springer, 1990).

Dr. Nijmeijer is a member of the editorial board of the SIAM Journal on Control and Optimization. 